\title{
LINC00899 promotes osteogenic differentiation by targeting miR-374a and RUNX2 expression
}

\author{
XIAOYA GAO, YUN XUE and KECHUN YANG \\ Department of Endocrinology, The Affiliated Changzhou No. 2 People's Hospital of \\ Nanjing Medical University, Changzhou, Jiangsu 213161, P.R. China \\ Received April 26, 2020; Accepted May 10, 2021
}

DOI: $10.3892 /$ etm.2021.10505

\begin{abstract}
Accumulating researches indicate that long non-coding RNAs (lncRNAs) participate in human bone mesenchymal stem cells (hBMSCs) osteogenic differentiation. The present study aimed to investigate the underlying molecular mechanisms of long intergenic non-protein coding RNA 899 (LINC00899) in osteoporosis. Therefore, reverse transcription-quantitative PCR was performed to evaluate the expression levels of LINC00899, microRNA (miR)-374a and runt-related transcription factor 2 (RUNX2) in clinical tissues and hBMSCs. The potential interaction between miR-374a and LINC00899 or RUNX2 was predicted utilizing the StarBase software and verified by luciferase reporter and RNA binding protein immunoprecipitation assays. In addition, alkaline phosphatase activity and Alizarin Red $\mathrm{S}$ staining were used to evaluate the osteogenic potential of hBMSCs. The results showed that the expression levels of LINC00899 were gradually increased, whilst those of miR-374a were decreased as the osteogenic induction process progresses. Additionally, the expression of LINC00899 was downregulated in the bone tissues of patients with osteoporosis, where LINC00899 knockdown reduced the expression levels of osteogenesis-related genes osteocalcin (OCN), osteopontin (OPN) and RUNX2 in hBMSCs. LINC00899 was also found to directly target miR-374a, thereby inhibiting its expression. Finally, it was predicted that RUNX2 could be directly targeted by miR-374a, such that miR-374a silencing partially abolished the inhibitory effect of LINC00899 knockdown on
\end{abstract}

Correspondence to: Dr Kechun Yang, Department of Endocrinology, The Affiliated Changzhou No. 2 People's Hospital of Nanjing Medical University, 68 Gehu Road, Changzhou, Jiangsu 213161, P.R. China

E-mail: kechunyang820422@163.com

Abbreviations: LncRNAs, long noncoding RNAs; hBMSCs, human bone mesenchymal stem cells; RT-qPCR, reverse transcription-quantitative PCR; RIP, RNA immunoprecipitation; miRNAs, microRNAs

Key words: long intergenic non-protein coding RNA 899, osteoporosis, microRNA-374a, runt-related transcription factor 2 the expression of RUNX2, OPN and OCN. Overall, findings of the present study suggested that LINC00899 could facilitate the osteogenic differentiation of hBMSCs and prevent osteoporosis by sponging miR-374a to enhance the expression of RUNX2, which provide a potentially useful therapeutic strategy for patients with osteoporosis.

\section{Introduction}

Osteoporosis is a common systemic bone disease particularly in the older population that is characterized by diminished bone density and mass, coupled with destruction of the bone microstructure $(1,2)$. Human bone mesenchymal stem cells (hBMSCs) are multipotent progenitor cells that have the potential to differentiate into osteoblasts, which serve critical roles in bone formation (3). It has been previously reported that recovery of hBMSC osteogenic differentiation ability may attenuate bone loss in osteoporosis (4-6). Therefore, directional differentiation of hBMSCs may be considered to be a potential therapeutic strategy for osteoporosis.

Long non-coding RNAs (lncRNAs) are a class of non-protein coding RNAs that are $>200$ nucleotides (nt) in length $(7,8)$. Increasing evidence has indicated that IncRNAs are involved in the occurrence and development of a number of diseases, including osteoporosis (9). Jiang et al (10) reported that the IncRNA small nucleolar RNA host gene could inhibit the osteogenic differentiation of BMSCs by negatively regulating the p38 MAPK signaling pathway. In addition, Chen et al (11) demonstrated that bone marrow stem cell-related lncRNA alleviated the progression of osteoporosis by inhibiting osteoclast differentiation. A study reported that the expression of long intergenic non-protein coding RNA 899 (LINC00899) was upregulated in the serum and bone marrow of patients with acute myeloid leukemia, suggesting that LINC00899 expression may be a potential biomarker for the diagnosis and prognosis of acute myeloid leukemia (12). However, the molecular mechanism underlying the role of LINC00899 in the occurrence and development of osteoporosis remain to be fully explored. Furthermore, lncRNAs may act as competitive endogenous RNAs that sponge microRNAs (miRNAs or miRs), thereby regulating osteogenic differentiation. For example, depletion of the lncRNA KCNQ1 opposite strand/antisense transcript 1 (KCNQ1OT1) was found to impede osteogenic differentiation by regulating miR-214 (13). 
Additionally, lncRNA MSC-antisense 1 could upregulate bone morphogenetic protein (BMP)2 expression to modulate osteogenic differentiation by sponging miR-140-5p (14). Therefore, the present study aimed to investigate whether LINC00899 could affect the progression of osteoporosis by regulating the expression of downstream miRNAs.

miRNAs are short non-coding RNAs with a length of $\sim 22 \mathrm{nt}$, which serve as vital regulators in a number of biological processes, including cell proliferation and differentiation (15). It has been reported that some miRNAs are involved in osteoporosis by binding to the 3'-untranslated regions (3'-UTR) of their target mRNAs (16). For example, Wang et al (17) showed that miR-765 exerted a potential role in attenuating osteogenic differentiation by targeting BMP6. Recently, Zhang et al (18) demonstrated that miR-664-5p could promote the osteogenic differentiation by direct targeting the high mobility group A2 protein. Additionally, Li et al (19) showed that miR-291a-3p improved cell viability and promoted osteogenic differentiation by regulating Dickkopf 1 . In another study, Li et al (20) suggested that miR-374a was involved in the progression of osteosarcoma (OS) and facilitated OS cell migration by regulating $\mathrm{Wnt} / \beta$-catenin signaling. However, the biological role of miR-374a in osteoporosis remains unclear.

It has been suggested that runt-related transcription factor 2 (RUNX2) is indispensable for bone formation (21) and a potent inducer of osteogenic differentiation (22). In addition, RUNX2 was identified as a downstream target of several miRNAs, such as miR-21 (23), miR-365a (24) and miR-217 (25). Therefore, the present study hypothesized that miR-374a may regulate osteoporosis progression by regulating RUNX2. The present study was undertaken to determine the biological role of LINC00899 in osteoporosis and investigated whether LINC00899 could sponge miR-374a to enhance RUNX2 expression, with the aim of providing a novel therapeutic strategy for alleviating osteoporosis.

\section{Materials and methods}

Clinical samples. Bone tissues $(100 \mathrm{mg})$ were collected from patients with osteoporosis $(n=15$; female, 7; male, 8; age, 51-74 years) and healthy controls $(n=15$; female, 7; male, 8; age, 50-73 years) at the Affiliated Changzhou No. 2 People's Hospital of Nanjing Medical University (Nanjing, China) between March 2016 and April 2018. The inclusion criteria for patients were as follows: i) Patients were diagnosed with osteoporosis, and ii) patients provided informed consent. Patients with complications due to other diseases, such as chronic inflammatory diseases, such as rheumatoid arthritis and inflammatory bowel disease were excluded. The bone fragments extracted from the transcervical region of the femoral neck were dissected into smaller fragments, washed three times with PBS and stored at $-80^{\circ} \mathrm{C}$ until further analysis. Written informed consent was obtained from all patients prior to study enrollment. The present study was approved by the Ethics Committee of the Affiliated Changzhou No. 2 People's Hospital of the Nanjing Medical University.

Cell culture and induction of osteogenic differentiation. 293 T cells were purchased from The Cell Bank of Type Culture Collection of The Chinese Academy of Sciences. Cells were cultured in RPMI-1640 medium (Gibco; Thermo Fisher Scientific, Inc.) supplemented with 10\% FBS (Gibco; Thermo Fisher Scientific, Inc.) and 1\% penicillin-streptomycin (Thermo Fisher Scientific,Inc.), and maintained in a humidified atmosphere containing $5 \% \mathrm{CO}_{2}$ at $37^{\circ} \mathrm{C}$. The hBMSCs were obtained from the BeNa Culture Collection; Beijing Beina Chunglian Biotechnology Research Institute (cat. no. BNCC100385; http://www.bnbio.com/pro/pl/6/p_242206.html) and cultured in $\alpha$-minimum essential medium ( $\alpha$-MEM; Gibco; Thermo Fisher Scientific Inc.) supplemented with $10 \%$ fetal bovine serum (FBS; Gibco; Thermo Fisher Scientific, Inc.), $100 \mathrm{mg} / \mathrm{ml}$ penicillin and $100 \mathrm{U} / \mathrm{ml}$ streptomycin at $37^{\circ} \mathrm{C}$ in a humidified incubator containing $5 \% \mathrm{CO}_{2}$. To induce osteogenic differentiation, cells were treated with $10 \mu \mathrm{mol} / 1$ dexamethasone, $200 \mu \mathrm{M}$ ascorbic acid and $10 \mathrm{mmol} / 1 \beta$-glycerophosphate (Sigma-Aldrich; Merck $\mathrm{KGaA})$ at $37^{\circ} \mathrm{C}$ in $5 \% \mathrm{CO}_{2}$ and the medium was changed every 3 days (26).

Cell transfection. The short hairpin RNA (shRNA) targeting LINC00899 (5'-GCACAUGAGAUCGACUGACUA-3') was cloned into the pGHP1/Neo vector (Shanghai GenePharma Co., Ltd.) to generate shLINC00899. miR-374a mimics (5'-CUUAUCAGAUUGUAUUGUAAUU-3'), miR-374a inhibitor (5'-CACUUAUCAGGUUGUAUUAUAA-3'), and their corresponding negative controls (shNC; NC mimics, 5'-UCA CAACCUCCUAGAAAGAGUAGA-3'; NC inhibitor, 5'-UAA GUACAAUAAUUGCGCCACU-3') were purchased from Shanghai GenePharma Co., Ltd. To overexpress LINC00899, the full-length LINC00899 sequence was sub-cloned into the pcDNA3.1 vector (Shanghai GenePharma Co., Ltd). Cell were transfected with $50 \mathrm{nM}$ shLINC00899, $50 \mathrm{nM}$ shNC, $50 \mathrm{nM}$ miR-374a mimics, $50 \mathrm{nM}$ miR-374a inhibitor, $50 \mathrm{nM} \mathrm{NC}$ mimics or $50 \mathrm{nM}$ NC inhibitor using Lipofectamine ${ }^{\circledR} 2000$ (Invitrogen; Thermo Fisher Scientific, Inc.) for $48 \mathrm{~h}$ prior to subsequent experiments.

Bioinformatic analysis and dual-luciferase reporter assay. The StarBase 2.0 database (http://starbase.sysu.edu.cn/) was used to predict the binding sites between miR-374a and LINC00899 or RUNX2. Mutants within the miR-374a binding site were created using the QuikChange II Site Directed Mutagenesis kit (Agilent Technologies, Inc.). The wild-type (WT) or mutant (Mut) sequences of the miR-374a binding site in the 3'-UTR of LINC00899 and RUNX2 were cloned into a pmirGLO reporter vector (Shanghai GenePharma Co., Ltd.). Subsequently, 293T cells were co-transfected with $100 \mathrm{nM}$ miR-374a mimics or NC mimics and 0.6 $\mu \mathrm{g}$ pmirGLO-LINC00899-WT/Mut or pmirGLO-RUNX2-WT/Mut. Following incubation for $48 \mathrm{~h}$, the relative luciferase activity was detected using a dual-luciferase reporter assay system (Promega Corporation). Firefly luciferase activity was normalized to Renilla luciferase activity.

Reverse transcription-quantitative PCR (RT-qPCR) analysis. Total RNA was extracted from tissues and hBMSCs using TRIzol $^{\circledR}$ reagent (Invitrogen; Thermo Fisher Scientific, Inc.) according to the manufacturer's protocols. PrimeScript ${ }^{\mathrm{TM}}$ RT reagent kit (Takara Bio, Inc.) was used for cDNA generation of LINC00899 and mRNA, using the following reaction conditions: $42^{\circ} \mathrm{C}$ for $15 \mathrm{~min}$ followed by 3 cycles at $85^{\circ} \mathrm{C}$ 
for 5 sec. For miR-374a, a TaqMan ${ }^{\mathrm{TM}}$ MicroRNA Reverse Transcription kit (Thermo Fisher Scientific, Inc.) was used for cDNA generation using the following reaction conditions: $50^{\circ} \mathrm{C}$ for $5 \mathrm{~min}$ and $80^{\circ} \mathrm{C}$ for $2 \mathrm{~min}$. qPCR was performed on the ABI 7900 Detection System (Applied Biosystems; Thermo Fisher Scientific, Inc.) using the SYBR-Green PCR Master Mix kit (Thermo Fisher Scientific, Inc.). The thermocycling conditions were as follows: Pre-denaturation at $95^{\circ} \mathrm{C}$ for $1 \mathrm{~min}$, followed by 40 cycles of $95^{\circ} \mathrm{C}$ for $15 \mathrm{sec}, 60^{\circ} \mathrm{C}$ for $30 \mathrm{sec}$ and $72^{\circ} \mathrm{C}$ for $30 \mathrm{sec}$. The data were analyzed using the $2^{-\Delta \Delta \mathrm{Cq}}$ method (27). The primer sequences were as follows: LINC00899 forward, 5'-CAGTCAGCCTCAGTTTCCAA-3' and reverse, 5'-AGG CAGGGCTGTGCTGAT-3'; miR-374a forward, 5'-GGTCAC AGTGAACCGGTC-3' and reverse, 5'-GTGCAGGGTCCG AGGT-3'; RUNX2 forward, 5'-CTTATACAATGTCAACAG CC-3' and reverse, 5'-TCCTTATGCTCTTTCTTCC-3'; OPN forward, 5'-CAAATACCCAGATGCTGTGGC-3' and reverse, 5'-TCCTGGCTGTCCACATGGTC-3'; OCN forward, 5'-GCC GAGAAATGTTGGAGAAA-3' and reverse, 5'-CTCCTTAAT CTGGCCAACCA-3'; GAPDH forward, 5'-CCACTCCTC CACCTTTGAC-3' and reverse, 5'-ACCCTGTTGCTGTAG CCA-3' and U6 forward, 5'-CTTCGGCAGCACATATAC T-3' and reverse, 5'-AAAATATGGAACGCTTCACG-3'. The expression of LINC00899, RUNX2, OCN and OPN expression was normalized to the internal reference gene GAPDH, whereas that of miR-374a expression was normalized to U6.

RNA immunoprecipitation (RIP) assay. RIP assay was performed using the Magna RNA-binding protein immunoprecipitation kit (EMD Millipore). Briefly, hBMSCs were lysed with $100 \mu 1$ Lysis Buffer (EMD Millipore) for $5 \mathrm{~min}$ at $4^{\circ} \mathrm{C}$. Argonaute 2 (Ago2; $5 \mu \mathrm{g}$; cat. no. ab32381; Abcam.) and $\operatorname{IgG}(5 \mu \mathrm{g}$; cat. no. ab172730; Abcam) were incubated with $50 \mu \mathrm{l} \mathrm{A} / \mathrm{G}$ magnetic beads for $1 \mathrm{~h}$ at $4^{\circ} \mathrm{C}$. Then, hBMSCs cell lysates was mixed with the beads to incubate for $4 \mathrm{~h}$ at $4^{\circ} \mathrm{C}$. RNA was extracted from the immunoprecipitation complex using $50 \mu \mathrm{g} / \mathrm{ml}$ Proteinase $\mathrm{K}$ (cat. no. P2308; Sigma-Aldrich; Merck KGaA) at $55^{\circ} \mathrm{C}$ for $30 \mathrm{~min}$ to digest the protein. Subsequently, the enrichment of LINC00899 and miR-374a was measured by RT-qPCR.

Alkaline phosphatase (ALP) activity. The ALP activity was measured at 0,7 and 14 days following cell culture, using the ALP assay kit (cat. no. P0321; Beyotime Institute of Biotechnology) according to the manufacturer's protocol. Briefly, hBMSCs $\left(1 \times 10^{5}\right)$ were collected, rinsed with PBS, incubated and lysed with RIPA buffer (Beyotime Institute of Biotechnology). The cell lysate was centrifuged at $15,000 \mathrm{x} \mathrm{g}$ at $4^{\circ} \mathrm{C}$ for $8 \mathrm{~min}$ before ALP activity was determined in the supernatant. The optical absorbance was finally measured at $450 \mathrm{~nm}$ using a microplate reader (Thermo Fisher Scientific, Inc.).

Alizarin Red $S(A R S)$ staining. Following osteogenic differentiation, treated hBMSCs $\left(1 \times 10^{5}\right)$ were washed twice with PBS and then fixed with $4 \%$ paraformaldehyde for $20 \mathrm{~min}$ at room temperature. Subsequently, the cells were washed twice with PBS and stained with $1 \mathrm{ml}$ ARS (Sigma-Aldrich; Merck $\mathrm{KGaA}$ ) at $37^{\circ} \mathrm{C}$ for $10 \mathrm{~min}$. After washing with distilled water, images of the cells were captured under a light microscope (magnification, x200; Olympus Corporation).
Western blot analysis. Total proteins were extracted from hBMSCs using a RIPA buffer (Beyotime Institute of Biotechnology) and quantified using the (BCA) Protein Assay kit (Pierce; Thermo Fisher Scientific, Inc.). The protein samples $(20 \mu \mathrm{g}$ g/lane) were separated by $10 \%$ SDS-PAGE and transferred onto PVDF membranes. Following blocking for $2 \mathrm{~h}$ with $5 \%$ non-fat dry milk at room temperature, the membranes were incubated with antibodies against RUNX2 (1:1,000; cat. no. 12556; Cell Signaling Technology, Inc.), osteopontin (OPN; 1:1,000; cat. no. ab214050; Abcam), osteocalcin (OCN; 1:1,000; cat. no. ab93876; Abcam) and GAPDH (1:1,000; cat. no. ab8245; Abcam) at $4^{\circ} \mathrm{C}$ overnight. The next day, the membranes were incubated with a horseradish peroxidase-conjugated secondary antibody (1:1,000; cat. no. ab205718; Abcam) for $1 \mathrm{~h}$ at $37^{\circ} \mathrm{C}$. Finally, the protein bands were visualized using the Pierce ${ }^{\mathrm{TM}} \mathrm{ECL}$ Western Blotting substrate (Thermo Fisher Scientific, Inc.).

Statistical analysis. All experiments were repeated $\geq$ three times. The data were analyzed using the GraphPad Prism 6.0 software (GraphPad Software, Inc.) and presented as the mean \pm standard deviation. The differences between two groups were analyzed using unpaired Student's t-test, whilst those among multiple groups were compared using one-way ANOVA followed by Tukey's post hoc test. $\mathrm{P}<0.05$ was considered to indicate a statistically different difference.

\section{Results}

Expression levels of LINC00899 and miR-374a during osteogenic differentiation. The dynamic expression pattern of LINC00899 and miR-374a was determined on days 0, 7 and 14 after osteogenic differentiation. As shown in Fig. 1A and B, the expression levels of LINC00899 were increased in a time dependent manner, whilst those of miR-374a were decreased as the osteogenic induction process progressed. Furthermore, RT-qPCR and western blot analysis revealed that the mRNA and protein expression levels of RUNX2, OPN and OCN were also gradually increased during the process of osteogenic differentiation in a time dependent manner (Fig. 1C-F). In addition, ALP activity and the area of mineralized nodules were markedly increased during osteogenic induction, as shown by ALP assay and ARS, respectively (Fig. 1G and H). These aforementioned results suggest that LINC00899 expression was upregulated whereas miR-374a expression was downregulated during osteogenic differentiation.

Knockdown of LINC00899 promotes the progression of osteoporosis. RT-qPCR assay results demonstrated that the LINC00899 expression levels were significantly higher in non-osteoporotic tissues compared with those in tissues from patients with osteoporosis (Fig. 2A). Subsequently, to investigate the effect of LINC00899 on osteogenic differentiation, LINC00899 expression was knocked down in hBMSCs on day 14 following osteogenic induction using shRNA, the transfection efficiency of which was confirmed by RT-qPCR (Fig. 2B). Subsequently, LINC00899 knockdown was found to have downregulated the expression of osteogenesis-related genes, RUNX2, OPN and OCN, at both mRNA and protein levels (Fig. 2C-F). In addition, results 
A

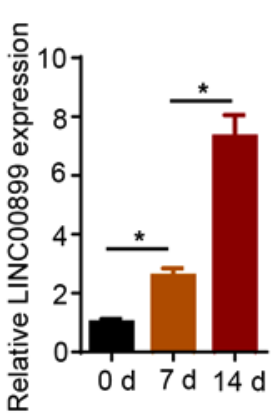

B

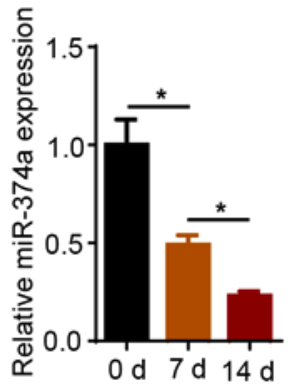

$\mathrm{C}$

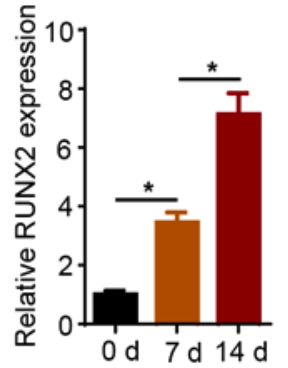

D

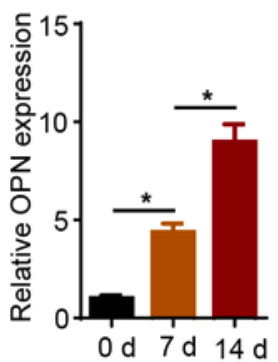

E

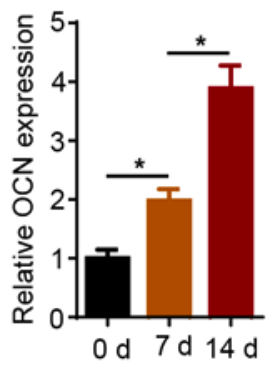

$\mathrm{F}$

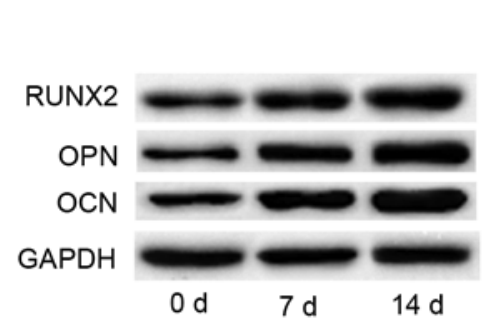

G

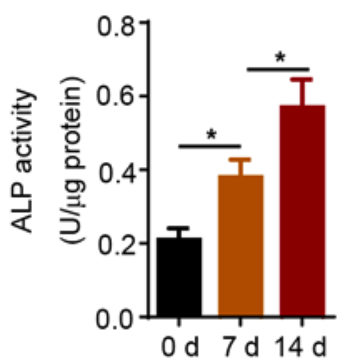

0 d 7 d $14 d$
$\mathrm{H}$

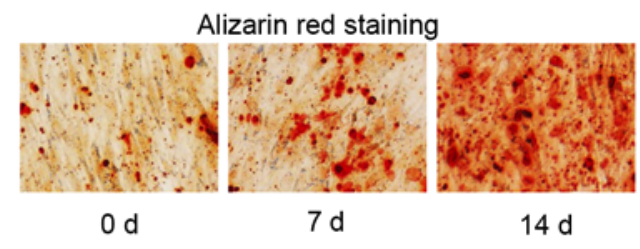

Figure 1. Expression levels of LINC00899 and miR-374a during osteogenic differentiation. Expression levels of (A) LINC00899 and (B) miR-374a in hBMSCs treated with osteogenic differentiation medium for 0,7 and 14 days as detected by RT-qPCR. RT-qPCR was used to detect the mRNA levels of (C) RUNX2, (D) OPN and (E) OCN during osteoblast differentiation of hBMSCs. (F) Western blotting was used to measure the protein levels of RUNX2, OPN and OCN during osteoblast differentiation of hBMSCs. (G) ALP activity and (H) Alizarin red S staining were detected on 0,7 and 14 days after osteogenic induction (magnification, $\mathrm{x} 200$ ). The data were presented as mean \pm SD. ${ }^{*} \mathrm{P}<0.05$. LINC00899, long intergenic non-protein coding RNA 899; miR, microRNA; RUNX2, runt-related transcription factor 2; OPN, osteopontin; OCN, osteocalcin; RT-qPCR, reverse transcription-quantitative PCR; hBMSCs, human bone mesenchymal stem cells; ALP, alkaline phosphatase.

from ALP assay demonstrated that LINC00899 knockdown significantly decreased ALP activity compared with that in cells transfected with shNC (Fig. 2G). ARS staining revealed that the number of mineralized nodules was reduced following transfection of hBMSCs with shLINC00899 (Fig. 2H). Taken together, these findings suggest that LINC00899 knockdown reduced the expression of osteogenesis-related genes, ALP activity and osteogenic capacity of hBMSCs.

miR-374a is a target of LINC00899. A potential binding site for miR-374a on LINC00899 was predicted using the StarBase software (Fig. 3A). miR-374a expression was increased in hBMSCs following transfection with miR-374a mimics compared with that in cells transfected with NC mimics (Fig. 3B). Data from dual-luciferase reporter assays revealed that miR-374a mimic transfection reduced the luciferase activity of LINC00899-WT, but not on the LINC00899-Mut plasmid (Fig. 3C). RIP assay showed that LINC00899 and miR-374a were significantly enriched in samples pulled down by Ago2-containing beads compared with those pulled down by the IgG-containing beads (Fig. 3D). Furthermore, the expression levels of miR-374a were measured in non-osteoporotic and osteoporotic tissues, where the results showed that the miR-374a expression level was significantly lower in non-osteoporotic tissues compared with that in osteoporotic tissues (Fig. 3E). RT-qPCR confirmed that LINC00899 was upregulated in hBMSCs transfected with the LINC00899 overexpression plasmid compared with that in cells transfected with the empty plasmid (Fig. 3F). Subsequently, LINC00899 knockdown was found to significantly increase the expression of miR-374a, whilst LINC00899 overexpression exerted the opposite effect (Fig. 3G). Overall, these results indicated that LINC00899 could directly interact with and inhibit miR-374a expression.

LINC00899 regulates osteogenic differentiation by inhibiting miR-374a expression. Bioinformatics analysis using the Starbase software predicted that RUNX2 could be a potential target of miR-374a (Fig. 4A). Subsequently, dual-luciferase reporter assay verified that transfection with the miR-374a mimics significantly attenuated the luciferase activity of RUNX2-WT (Fig. 4B). However, miR-374a overexpression exerted no significant effect on the luciferase activity of RUNX2-Mut (Fig. 4B). miR-374a expression was significantly downregulated following the transfection of miR-374a inhibitor into hBMSCs (Fig. 4C). In addition, silencing of miR-374a significantly increased the expression of RUNX2 (Fig. 4D). To further investigate whether LINC00899 could regulate the expression of RUNX2 via miR-374a, hBMSCs were transfected with shNC, shLINC00899 or shLINC00899 + miR-374a inhibitor. RT-qPCR and western blot analysis showed that LINC00899 knockdown downregulated RUNX2 expression, but the simultaneous inhibition of miR-374 expression significantly reversed this effect (Fig. 4E and F). Furthermore, co-transfection of hBMSCs with the miR-374a inhibitor partially abolished the suppressive effects of LINC00899 knockdown on the expression of OPN and OCN (Fig. 4G-I). Results from the ALP activity assay 
A
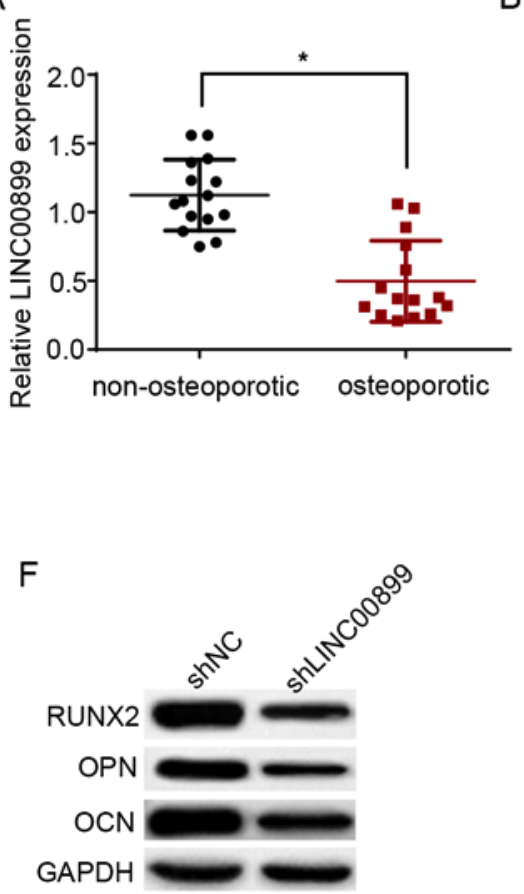

B

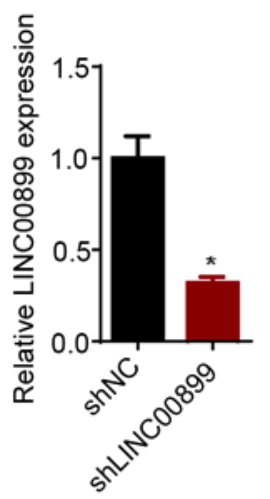

G

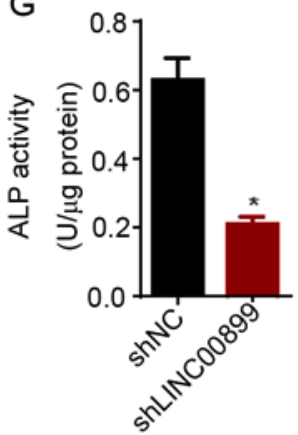

C

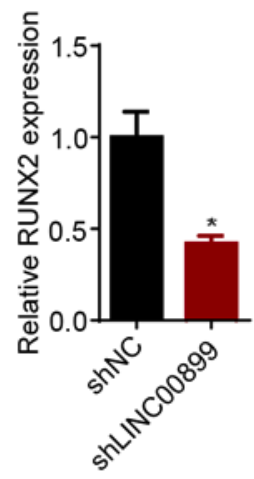

E
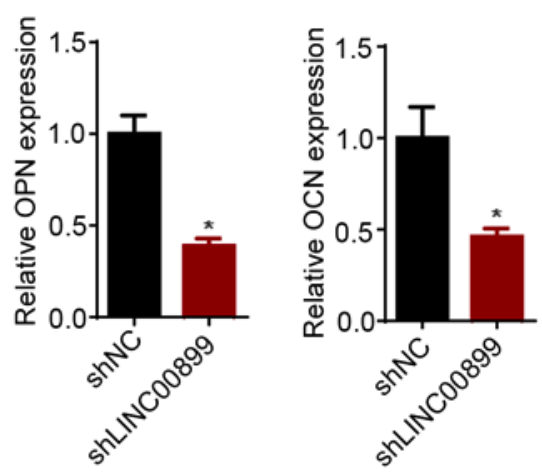

$\mathrm{H}$

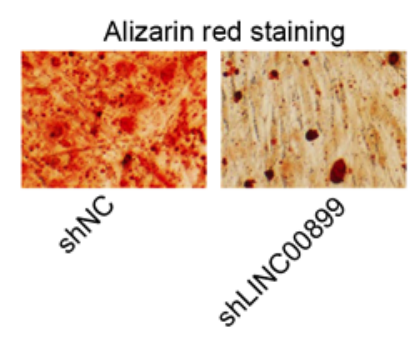

Figure 2. Knockdown of LINC00899 expression promotes the progression of osteoporosis. (A) RT-qPCR was used to detect the expression of LINC00899 in femoral bone tissues from patients with osteoporosis and health individuals. ${ }^{*} \mathrm{P}<0.05$. (B) Expression of LINC00899 in hBMSCs transfected with shNC and shLINC00899 was detected by RT-qPCR. mRNA expression of osteogenesis-related genes (C) RUNX2, (D) OPN and (E) OCN in hBMSCs transfected with shNC and shLINC00899 was detected by RT-qPCR. (F) Western blotting was utilized to detect the protein levels of RUNX2, OPN and OCN in hBMSCs transfected with shNC and shLINC00899. (G) ALP activity was detected in hBMSCs transfected with shNC and shLINC00899. (H) Alizarin red S staining was measured in hBMSCs transfected with shNC and shLINC00899 (magnification, $\mathrm{x} 200$ ). The data were presented as mean \pm SD. ${ }^{*}<0.05$ vs. shNC. RT-qPCR, reverse transcription-quantitative PCR; LINC00899, long intergenic non-protein coding RNA 899; hBMSCs, human bone mesenchymal stem cells; ALP, alkaline phosphatase; sh, short hairpin; NC, negative control; RUNX2, runt-related transcription factor 2; OPN, osteopontin; OCN, osteocalcin.

further verified that miR-374a silencing could reverse the inhibitory effects of LINC00899 knockdown on ALP activity (Fig. 4J). Additionally, ARS staining showed that miR-374a inhibitor could markedly restore the osteogenic capacity of hBMSCs transfected with shLINC00899 (Fig. 4K). These observations suggest that LINC00899 can upregulate RUNX2 expression by sponging miR-374a to alleviate osteoporosis.

\section{Discussion}

The present study demonstrated that LINC00899 could facilitate osteogenic differentiation and prevent osteoporosis by regulating the miR-374a/RUNX2 axis. Specifically, LINC00899 downregulated miR-374a expression, which could in turn directly target the 3'-UTR of RUNX2, thus downregulating its expression.

Emerging evidence has suggested that lncRNAs can serve an important role in bone diseases, such as osteoporosis, osteoarthritis, and osteosarcoma $(28,29)$. Han et al (30) revealed that downregulation of IncRNA taurine upregulated 1 could effectively inhibit osteoclast proliferation and serve as a potential target for the treatment of osteoporosis. Shen et al (31) demonstrated that IncRNA hox transcript antisense RNA (HOTAIR) was highly expressed in patients with osteoporosis, which prevents osteogenic differentiation by regulating the
Wnt/ $\beta$-catenin signaling pathway. In terms of LINC00899, Zhou et al (32) previously showed that the upregulation of LINC00899 suppressed breast cancer progression by regulating the expression of miR-425. Additionally, Dong et al (33) revealed that the upregulation of LINC00899 can promote the progression of acute myeloid leukemia. However, the biological effects of LINC00899 in osteogenic differentiation and osteoporosis remain poorly understood. To the best of our knowledge, the present study was the first to report that LINC00899 expression is downregulated in the bone tissues of patients with osteoporosis. Furthermore, knocking down LINC00899 expression was found to decrease the expression of osteogenesis-related genes, inhibit ALP activity whilst reducing ARS accumulation, eventually leading to the inhibition of osteogenic differentiation and progression of osteoporosis.

Accumulating evidence has suggested that lncRNAs can serve as miRNA sponges to regulate osteogenic differentiation and osteoporosis progression. For example, Wang et al (13) demonstrated that the upregulation of IncRNA KCNQ1OT1 promoted osteogenic differentiation by sponging miR-214 to upregulate BMP2.Zhang et al (34) reported that the upregulation of nuclear paraspeckle assembly transcript 1 promoted BMP1 expression to regulate osteogenic differentiation in hBMSCs by sponging miR-29b-3p. In addition, it has been suggested that miRNAs are involved in the occurrence of bone 
A

Mutant LINC00899: 5' UUAUAUAUAUAACACGCGGGC 3' Wildtype LINC00899: 5' UUAUAUAUAUAACAUAUUAUA 3' || ||| | hsa-miR-374a-5p: 3' GUGAAUAGUCCAACAUAAUAU 5'
B

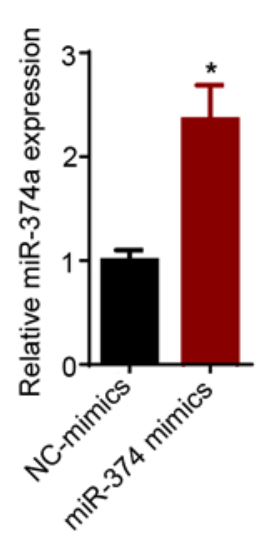

$E$
C

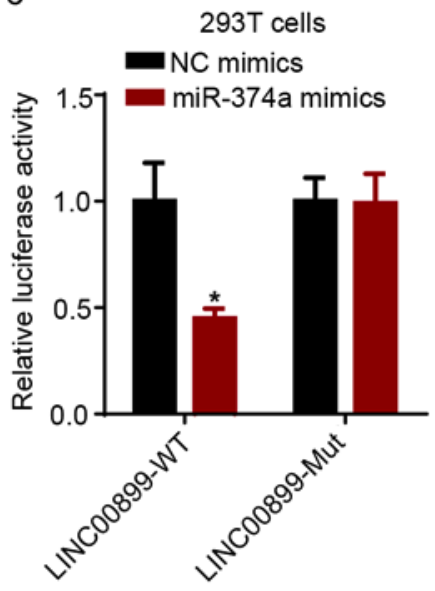

$\mathrm{F}$
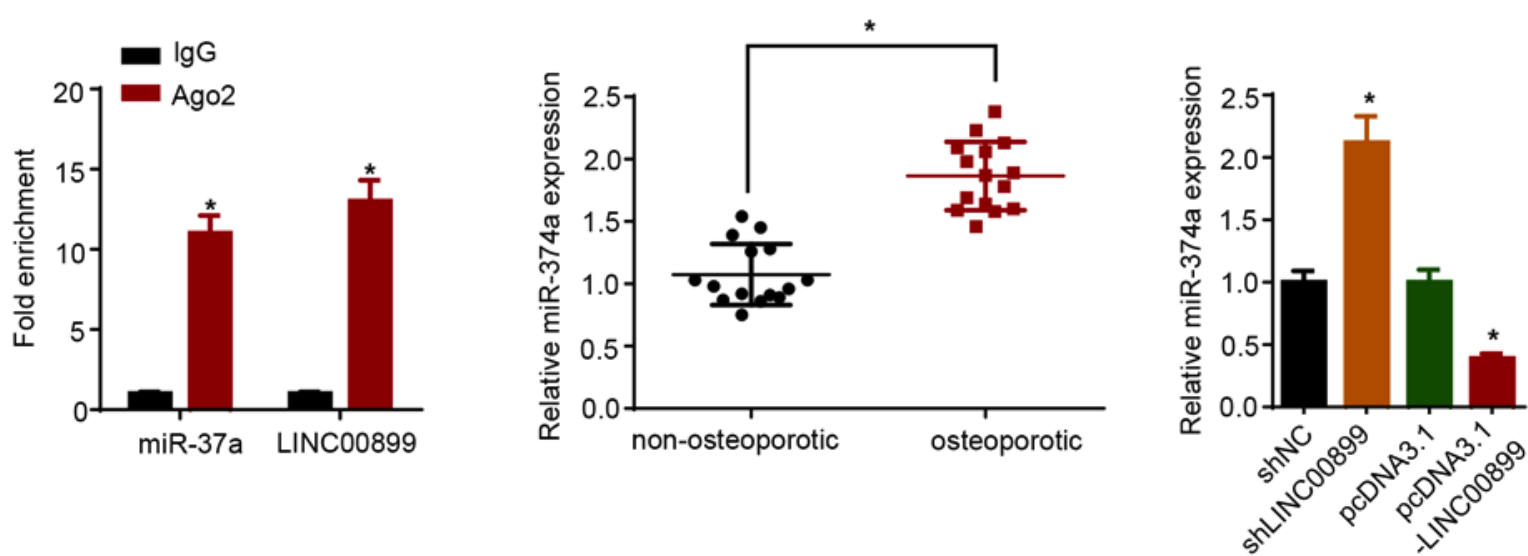

Figure 3. miR-374a is a target of LINC00899. (A) A potential binding site for miR-374a to LINC00899 was predicted by the starBase online tool. (B) The expression of miR-374a was detected by RT-qPCR in hBMSCs transfected with NC or miR-374a mimics. " $\mathrm{P}<0.05$ vs. NC mimics. (C) Dual-luciferase reporter assay was used to determine the luciferase activity of wild-type LINC00899 or mutant-type LINC00899 in 293 T cells. ${ }^{*} \mathrm{P}<0.05$ vs. NC mimics. (D) RNA immunoprecipitation assay was utilized to assess the potential interaction between miR-374 and LINC00899. "P<0.05 vs. IgG. (E) RT-qPCR used to detect the expression of miR-374a in femoral bone tissues from patients with osteoporosis and health individuals. "P<0.05. (F) The expression of LINC00899 in hBMSCs transfected with pcDNA3.1 or pcDNA3.1-LINC00899 was measured using RT-qPCR. "P<0.05 vs. pcDNA3.1. The data were presented as mean \pm SD. ${ }^{*} \mathrm{P}<0.05$. miR, microRNA; LINC00899, long intergenic non-protein coding RNA 899; RT-qPCR, reverse transcription-quantitative PCR; hBMSCs, human bone mesenchymal stem cells; sh, short hairpin; NC, negative control; Ago2, argonaute 2; WT, wild-type; Mut, mutant.

diseases, such as osteoporosis (35), osteoarthritis (36), and osteosarcoma (37). For example, Fan et al (38) demonstrated that miR-532-3p attenuated osteogenic differentiation by downregulating ETS-1. Additionally, Xiaoling et al (39) previously reported that the upregulation of miR-19b-3p could promote osteogenic differentiation in BMSCs. In the present study, miR-374a was predicted to be a potential target of LINC00899, which was verified by dual-luciferase reporter and RIP assays. Furthermore, the dynamic expression profile of LINC00899 and miR-374a during osteogenic differentiation was also determined. The results revealed that the expression of LINC00899 was gradually increased, whilst that of miR-374a was decreased, during osteogenic differentiation. These results suggest that miR-374a could be associated with osteoporosis progression by regulating osteogenic differentiation.

RUNX2 is widely recognized as an important transcription factor during osteogenic differentiation (40). Therefore, the regulatory mechanism of RUNX2 during osteogenic differentiation has attracted the attention of numerous research groups. Fu et al (41) showed that the upregulation of HOTAIRM1 promoted osteogenesis by modulating the activity of JNK and c-Jun to promote RUNX2 gene transcription. Another study by Chen et al (42) demonstrated that the upregulation of IncRNA AWPPH contributed to non-traumatic osteonecrosis by upregulating RUNX2. However, the mechanism associated with RUNX2 in osteoporosis has not been fully elucidated. Therefore, the present study aimed to actively explore the novel mechanisms by which RUNX2 may regulate osteogenic differentiation. RUNX2 was predicted as the direct target of miR-374a. RT-qPCR showed that RUNX2 was significantly upregulated during osteogenic differentiation and in hBMSCs transfected with the miR-374a inhibitor. In addition, depletion of LINC00899 inhibited the expression of osteogenesis-related markers RUNX2, OPN and OCN. This effect was counteracted following the co-transfection of hBMSCs with the miR-374a inhibitor. Furthermore, co-transfection with the miR-374a inhibitor neutralized the decrease in ALP activity and ARS accumulation induced by 
A

Mutant RUNX2: 5' CUGCAGCAAACAAGAGCCGCC 3' Wildtype RUNX2: 5' CUGCAGCAAACAAGAAUUAUA $3^{\prime}$ IIIIII hsa-miR-374a-5p: 3' GUGAAUAGUCCAACAUAAUAU 5' E

F
B

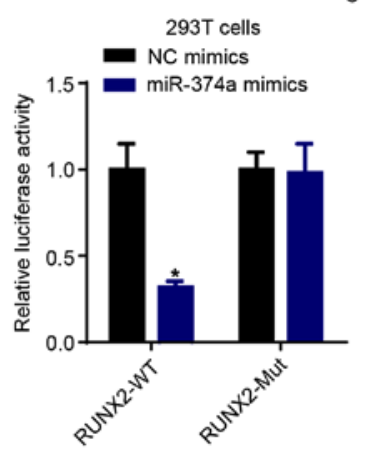

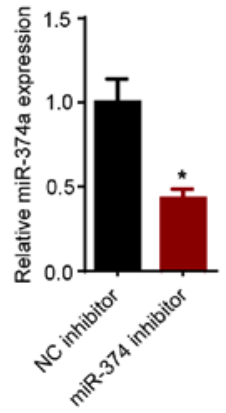
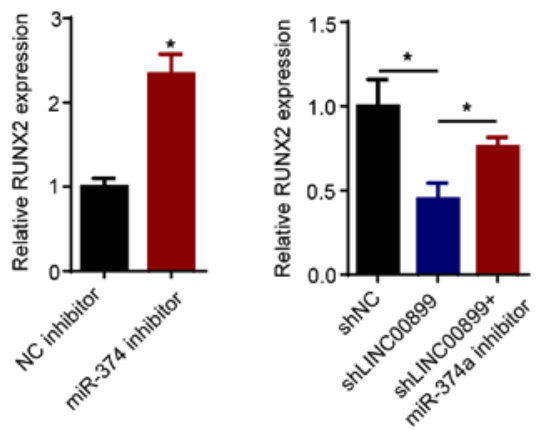

RUNX2

GAPDH
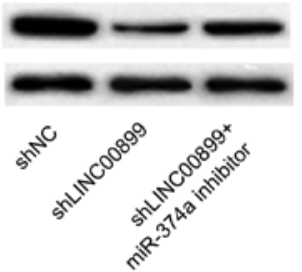

G

$\mathrm{H}$

I
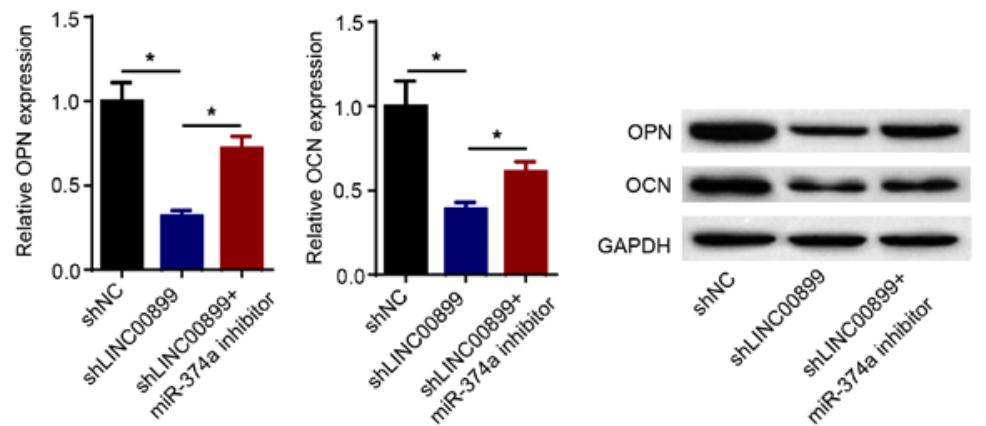

$\mathrm{K}$
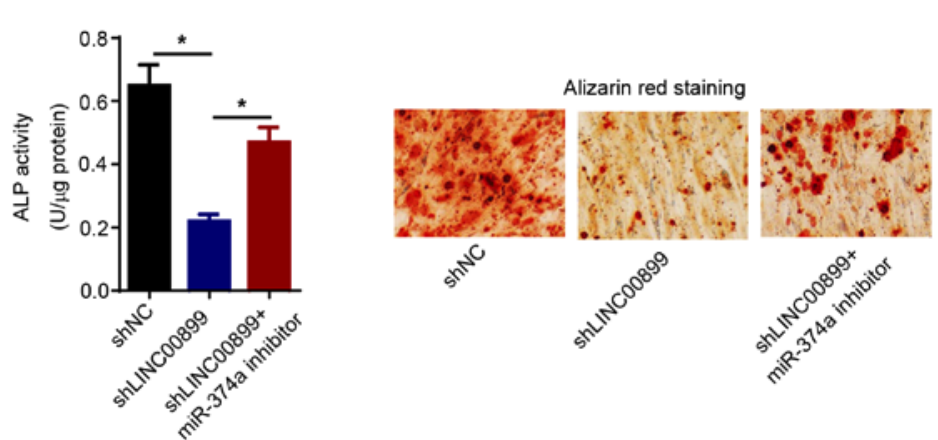

Figure 4. LINC00899 regulates osteogenic differentiation by suppressing miR-374a expression. (A) The predicted binding sites of miR-374a on the RUNX2 3'-untranslated region. (B) Dual-luciferase reporter assay was used to determine the luciferase activity of RUNX2-WT or RUNX2-Mut in 293T cells. "P<0.05 vs. NC mimics. (C) Expression of miR-374a was measured by RT-qPCR in hBMSCs transfected with NC inhibitor or miR-374a inhibitor. ${ }^{*}<<0.05$ vs. NC inhibitor. (D) The expression of RUNX2 in hBMSCs transfected with NC inhibitor and miR-374a inhibitor was detected by RT-qPCR. * $<0.05$ vs. NC inhibitor. (E) mRNA and (F) protein levels of RUNX2, mRNA levels of (G) OPN and (H) OCN, (I) protein levels of OPN and OCN were determined in hBMSCs transfected with shNC, shLINC00899, shLINC00899 + miR-374a inhibitor by RT-qPCR and western blotting. *P<0.05. (J) ALP activity was detected in hBMSCs transfected with shNC, shLINC00899 and shLINC00899 + miR-374a inhibitor. * $\mathrm{P}<0.05$. (K) Alizarin red S staining was detected in hBMSCs transfected with shNC, shLINC00899 and shLINC00899 + miR-374a inhibitor (magnification, $\mathrm{x} 200$ ). The data were presented as mean \pm SD. miR, microRNA; LINC00899, long intergenic non-protein coding RNA 899; RT-qPCR, reverse transcription-quantitative PCR; hBMSCs, human bone mesenchymal stem cells; sh, short hairpin; NC, negative control; WT, wild-type; Mut, mutant; ALP, alkaline phosphatase; RUNX2, runt-related transcription factor 2; OPN, osteopontin; OCN, osteocalcin. 
LINC00899 knockdown. LINC00899 also regulated RUNX2 expression by targeting miR-374a, supporting the regulatory effects of the LINC00899/miR-374a/RUNX2 axis during osteogenic differentiation.

In summary, the present study uncovered a role of LINC00899 in promoting osteogenic differentiation through the miR-374a/RUNX2 axis. Therefore, these findings may provide a novel theoretical basis for developing a treatment strategy for osteoporosis.

\section{Acknowledgements}

Not applicable.

\section{Funding}

No funding was received.

\section{Availability of data and materials}

The analyzed data sets generated during the study are available from the corresponding author on reasonable request.

\section{Authors' contributions}

XG and KY designed the study. XG, YX and KY performed the experiments, analyzed the data and prepared the figures. XG and KY drafted the initial manuscript. XG and KY authenticated the raw data in this study. All authors have read and approved the final manuscript.

\section{Ethics approval and consent to participate}

Ethical approval was obtained from the Ethics Committee of the Affiliated Changzhou No. 2 People's Hospital of Nanjing Medical University (Nanjing, China). All procedures performed in studies involving human participants were in accordance with the ethical standards of the institutional and national research committee. Written informed consent was obtained from all individual participants included in this study.

\section{Patient consent for publication}

Not applicable.

\section{Competing interests}

All authors declare that they have no competing interests.

\section{References}

1. Montalcini T, Romeo S, Ferro Y, Migliaccio V, Gazzaruso C and Pujia A: Osteoporosis in chronic inflammatory disease: The role of malnutrition. Endocrine 43: 59-64, 2013.

2. Kobayashi M, Sawada K, Yoshimura A, Yamamoto M, Shimizu A, Shimura K, Komura N, Miyamoto M, Ishida K and Kimura T: Clinical effects of switching from minodronate to denosumab treatment in patients with postmenopausal osteoporosis: A retrospective study. BMC Womens Health 20: 48, 2020.

3. Bianco P, Sacchetti B and Riminucci M: Stem cells in skeletal physiology and endocrine diseases of bone. Endocr Dev 21: 91-101, 2011.
4. Jing H, Liao L, An Y, Su X, Liu S, Shuai Y, Zhang X and Jin Y: Suppression of EZH2 prevents the shift of osteoporotic MSC fate to adipocyte and enhances bone formation during osteoporosis. Mol Ther 24: 217-229, 2016.

5. Li CJ, Cheng P, Liang MK, Chen YS, Lu Q, Wang JY, Xia ZY, Zhou HD, Cao X, Xie H, et al: MicroRNA-188 regulates age-related switch between osteoblast and adipocyte differentiation. J Clin Invest 125: 1509-1522, 2015.

6. Li Y, Fan L, Hu J, Zhang L, Liao L, Liu S, Wu D, Yang P, Shen L, Chen $\mathrm{J}$ and Jin Y: miR-26a rescues bone regeneration deficiency of mesenchymal stem cells derived from osteoporotic mice. Mol Ther 23: 1349-1357, 2015 .

7. Hu HL, Liu KY, Ram YI, Gao JL and Cao YM: Long noncoding RNA MIRG induces osteoclastogenesis and bone resorption in osteoporosis through negative regulation of miR-1897. Eur Rev Med Pharmacol Sci 23: 10195-10203, 2019.

8. Zeng X, Wang Y, Dong Q, Ma MX and Liu XD: DLX2 activates Wnt 1 transcription and mediates $W n t / \beta$-catenin signal to promote osteogenic differentiation of hBMSCs. Gene 744: 144564, 2020.

9. Gao GC, Yang DW and Liu W: LncRNA TERC alleviates the progression of osteoporosis by absorbing miRNA-217 to upregulate RUNX2. Eur Rev Med Pharmacol Sci 24: 526-534, 2020.

10. Jiang Y, Wu W, Jiao G, Chen Y and Liu H: LncRNA SNHG1 modulates p38 MAPK pathway through Nedd4 and thus inhibits osteogenic differentiation of bone marrow mesenchymal stem cells. Life Sci 228: 208-214, 2019.

11. Chen RS, Zhang XB, Zhu XT and Wang CS: LncRNA Bmncr alleviates the progression of osteoporosis by inhibiting RANML-induced osteoclast differentiation. Eur Rev Med Pharmacol Sci 23: 9199-9206, 2019.

12. Wang Y, Li Y, Song HQ and Sun GW: Long non-coding RNA LINC00899 as a novel serum biomarker for diagnosis and prognosis prediction of acute myeloid leukemia. Eur Rev Med Pharmacol Sci 22: 7364-7370, 2018.

13. Wang CG, Liao Z, Xiao H, Liu H, Hu YH, Liao QD and Zhong D: LncRNA KCNQ1OT1 promoted BMP2 expression to regulate osteogenic differentiation by sponging miRNA-214. Exp Mol Pathol 107: 77-84, 2019.

14. Zhang N, Hu X, He S, Ding W, Wang F, Zhao Y and Huang Z: LncRNA MSC-AS1 promotes osteogenic differentiation and alleviates osteoporosis through sponging microRNA-140-5p to upregulate BMP2. Biochem Biophys Res Commun 519: 790-796, 2019.

15. Ding J, Sha L, Shen P, Huang M, Cai Q and Li J: MicroRNA-18a inhibits cell growth and induces apoptosis in osteosarcoma by targeting MED27. Int J Oncol 53: 329-338, 2018.

16. Bellavia D, De Luca A, Carina V, Costa V, Raimondi L, Salamanna F, Alessandro R, Fini M and Giavaresi G: Deregulated miRNAs in bone health: Epigenetic roles in osteoporosis. Bone 122: 52-75, 2019.

17. Wang T, Zhang C, Wu C, Liu J, Yu H, Zhou X, Zhang J, Wang X, He S, Xu X, et al: miR-765 inhibits the osteogenic differentiation of human bone marrow mesenchymal stem cells by targeting BMP6 via regulating the BMP6/Smad1/5/9 signaling pathway. Stem Cell Res Ther 11: 62, 2020.

18. Zhang Y, Liu Y, Wu M, Wang H, Wu L, Xu B, Zhou W, Fan X, Shao J and Yang T: MicroRNA-664a-5p promotes osteogenic differentiation of human bone marrow-derived mesenchymal stem cells by directly downregulating HMGA2. Biochem Biophys Res Commun 521: 9-14, 2020.

19. Li ZH, Hu H, Zhang XY, Liu GD, Ran B, Zhang PG, Liao MM and $\mathrm{Wu}$ YC: miR-291a-3p regulates the BMSCs differentiation via targeting DKK1 in dexamethasone-induced osteoporosis. Kaohsiung J Med Sci 36: 35-42, 2020.

20. Li W, Meng Z, Zou T, Wang G, Su Y, Yao S and Sun X: miR-374a activates $\mathrm{Wnt} / \beta$-catenin signaling to promote osteosarcoma cell migration by targeting WIF-1. Pathol Oncol Res 26: 533-539, 2020.

21. Komori T: Requisite roles of Runx 2 and $\mathrm{Cbfb}$ in skeletal development. J Bone Miner Metab 21: 193-197, 2003.

22. Zhao Z, Zhao M, Xiao G and Franceschi RT: Gene transfer of the Runx 2 transcription factor enhances osteogenic activity of bone marrow stromal cells in vitro and in vivo. Mol Ther 12: 247-253, 2005.

23. Li L and Jiang D: Hypoxia-responsive miRNA-21-5p inhibits Runx2 suppression by targeting SMAD7 in MC3T3-E1 cells. J Cell Biochem 120: 16867-16875, 2019.

24. Cheng F, Yang MM and Yang RH: miRNA-365a-3p promotes the progression of osteoporosis by inhibiting osteogenic differentiation via targeting RUNX2. Eur Rev Med Pharmacol Sci 23: 7766-7774, 2019. 
25. Yang L, Zeng Z, Kang N, Yang JC, Wei X and Hai $Y$ : Circ-VANGL1 promotes the progression of osteoporosis by absorbing miRNA-217 to regulate RUNX2 expression. Eur Rev Med Pharmacol Sci 23: 949-957, 2019.

26. Kosmacheva SM, Volk MV, Yeustratenka TA, Severin IN and Potapnev MP: In vitro growth of human umbilical blood mesenchymal stem cells and their differentiation into chondrocytes and osteoblasts. Bull Exp Biol Med 145: 141-145, 2008.

27. Livak KJ and Schmittgen TD: Analysis of relative gene expression data using real-time quantitative PCR and the 2(-Delta Delta C(T)) method. Methods 25: 402-408, 2001.

28. Tong $X, G u$ PC, Xu SZ and Lin XJ: Long non-coding RNA-DANCR in human circulating monocytes: A potentia biomarker associated with postmenopausal osteoporosis. Biosci Biotechnol Biochem 79: 732-737, 2015.

29. Wang Q, Li Y, Zhang Y, Ma L, Lin L, Meng J, Jiang L, Wang L, Zhou P and Zhang Y: LncRNA MEG3 inhibited osteogenic differentiation of bone marrow mesenchymal stem cells from postmenopausal osteoporosis by targeting miR-133a-3p. Biomed Pharmacother 89: 1178-1186, 2017.

30. Han Y, Liu C, Lei M, Sun S, Zheng W, Niu Y and Xia X: LncRNA TUG1 was upregulated in osteoporosis and regulates the proliferation and apoptosis of osteoclasts. J Orthop Surg Res 14: 416, 2019.

31. Shen JJ, Zhang CH, Chen ZW, Wang ZX, Yang DC, Zhang FL and Feng KH: LncRNA HOTAIR inhibited osteogenic differentiation of BMSCs by regulating Wnt/beta-catenin pathway. Eur Rev Med Pharmacol Sci 23: 7232-7246, 2019.

32. Zhou W, Gong J, Chen Y, Chen J, Zhuang Q, Cao J, Mei Z and $\mathrm{Hu} \mathrm{B}$ : Long noncoding RNA LINC00899 suppresses breast cancer progression by inhibiting miR-425. Aging (Albany NY) 11: 10144-10153, 2019.

33. Dong X, Xu X and Guan Y:LncRNA LINC00899 promotes progression of acute myeloid leukaemia by modulating miR-744-3p/YY1 signalling. Cell Biochem Funct 38: 955-964, 2020.

34. Zhang Y, Chen B, Li D, Zhou X and Chen Z: LncRNA NEAT1/miR-29b-3p/BMP1 axis promotes osteogenic differentiation in human bone marrow-derived mesenchymal stem cells. Pathol Res Pract 215: 525-531, 2019.
35. Kelch S, Balmayor ER, Seeliger C, Vester H, Kirschke JS and van Griensven M: miRNAs in bone tissue correlate to bone mineral density and circulating miRNAs are gender independent in osteoporotic patients. Sci Rep 7: 15861, 2017.

36. Park S, Lee M, Chun CH and Jin EJ: The lncRNA, nespas, is associated with osteoarthritis progression and serves as a potential new prognostic biomarker. Cartilage 10: 148-156, 2019.

37. Delsin LEA, Roberto GM, Fedatto PF, Engel EE, Scrideli CA, Tone LG and Brassesco MS: Downregulated adhesion-associated microRNAs as prognostic predictors in childhood osteosarcoma. Pathol Oncol Res 25: 11-20, 2019.

38. Fan Q, Li Y, Sun Q, Jia Y, He C and Sun T: miR-532-3p inhibits osteogenic differentiation in MC3T3-E1 cells by downregulating ETS1. Biochem Biophys Res Commun 525: 498-504, 2020 .

39. Xiaoling G, Shuaibin L and Kailu L: MicroRNA-19b-3p promotes cell proliferation and osteogenic differentiation of BMSCs by interacting with lncRNA H19. BMC Med Genet 21: 11, 2020.

40. Yang GZ, Zhang WJ, Ding X, Zhang XK, Jiang XQ and Zhang ZY: Effect of overexpression of transcription factor Runx2 and Osterix on osteogenic differentiation of endothelial cells. Shanghai Kou Qiang Yi Xue 26: 353-357, 2017 (In Chinese).

41. Fu L, Peng S, Wu W, Ouyang Y, Tan D and Fu X: LncRNA HOTAIRM1 promotes osteogenesis by controlling JNK/AP-1 signalling-mediated RUNX2 expression. J Cell Mol Med 23: 7517-7524, 2019.

42. Chen X, Li J, Liang D, Zhang L and Wang Q: LncRNA AWPPH participates in the development of non-traumatic osteonecrosis of femoral head by upregulating Runx2. Exp Ther Med 19: $153-159,2020$.

This work is licensed under a Creative Commons Attribution-NonCommercial-NoDerivatives 4.0 International (CC BY-NC-ND 4.0) License. 